\title{
rDNA-Based Genotyping of Clinical Isolates of Candida albicans
}

\author{
URSZULA NAWROT, MAGDALENA PAJACZKOWSKA, KATARZYNA WŁODARCZYK, IZABELA MECLER
}

Department of Microbiology, Medical University of Wroclaw, Poland

Received 25 January 2010, revised 2 July 2010, accepted 7 July 2010

\begin{abstract}
The study presents an analysis of the restriction pattern of rDNA fragments of 95 C. albicans isolates previously classified on the basis of the presence of the intron in rDNA into genotypes A (62 isolates), B (28), and C (5). Most isolates (61) with genotype A were classified as "subtype a" and one as "subtype d" (Karahan and Akar; 2005). No differences were observed in the restriction patterns of the tested genotype B isolates. Similarly, most genotype C strains (4/5) showed the same restriction pattern. The results indicate low subtyping variations of the analyzed isolates, which is in contrast to published data obtained from a Turkish collection of yeasts.
\end{abstract}

K e y words: Candida albicans, group I self-splicing intron, rDNA genotyping, RFLP

Candida albicans is the most frequent fungal pathogen, being the causative agent of both superficial and deep-seated and life-threatening mycoses. Many molecular methods have been employed in investigating species evolution and epidemiology. One of them is the genotyping developed by Mercure et al. (1993) and McCullough et al. (1999a) based on the presence or absence of the self-splicing group I intron in the large subunit (CaLSU) of rRNA genes. Strains can be differentiated into three genotypes: genotype A without the intron, genotype B harboring the intron, and genotype $\mathrm{C}$ possessing LSU rDNA with and without the intron in a single genome. In the last decade, several authors reported the distribution of the A, B, and $\mathrm{C}$ genotypes in different groups of clinical C. albicans isolates (Tamura et al., 2001, Millar et al., 2002; Karahan, 2004; Karahan and Akar, 2005; Qi et al., 2005; Millar et al., 2005; Girish Kumar et al., 2006, Nawrot et al., 2004; Nawrot et al., 2008). Although the data obtained by the particular authors differ in detail, genotype A has been reported as prevalent in most groups of clinical isolates, including those regarded as invasive. It is supposed that intron-containing genotypes can be eliminated because of their high susceptibility to some drugs, for example 5-fluorocytosine, pentamidine, and bleomycin, interfering with the selfsplicing process (Mercure et al., 1993; Zhang et al., 2002; Jayaguru and Raghunathan, 2007). On the other hand, some authors observed increased occurrence of genotype C (McCullough et al., 1999b; Gurbuz and Kaleli, 2010). Recently, Karahan and Akar (2005) found significant differences in the LSU rDNA sequences of genotype A isolates and constructed an RFLP-based method for differentiating genotype A into eight subtypes. In this communication we present the results of the RFLP analysis of LSU rDNA of C. albicans isolates, which is complementary to our previous study on the distribution of CaLSU among C. albicans isolated from blood and the respiratory tract (Nawrot et al., 2008).

The study was performed on 95 isolates of C. albicans, including 55 blood isolates obtained from BCCM/ IHEM (30 genotype A, $20 \mathrm{~B}$, and $5 \mathrm{C}$ ) and 40 isolates (32 genotype A and $8 \mathrm{~B}$ ) from the laboratory collection of Wrocław Medical University and originating from different clinical samples (22 from sputum or pharyngeal swabs, 7 from blood, and 8 from body fluids or pus). Genomic DNA of the tested yeasts was extracted using the CTAB method according to O'Donnell et al. (1997). The PCR assay was performed with the primer pair CA-INT-L (5'-ATA AGG GAA GTC GGC AAA ATA GAT CCG TAA-3') and CAINT-R (5' CCT TGG CTG TGG TTT CGC TAG ATA GTA GAT-3'), described previously by McCullough et al. (1999b). DNA samples were denatured at $94^{\circ} \mathrm{C}$ for $3 \mathrm{~min}$ before 30 cycles of $94^{\circ} \mathrm{C}$ for $1 \mathrm{~min}, 65^{\circ} \mathrm{C}$

* Corresponding author: U. Nawrot, Department of Microbiology, Medical University of Wroclaw, Chałubinskiego 4, 50-368 Wrocław, Poland; e-mail: nawrot@mbio.am.wroc.pl 


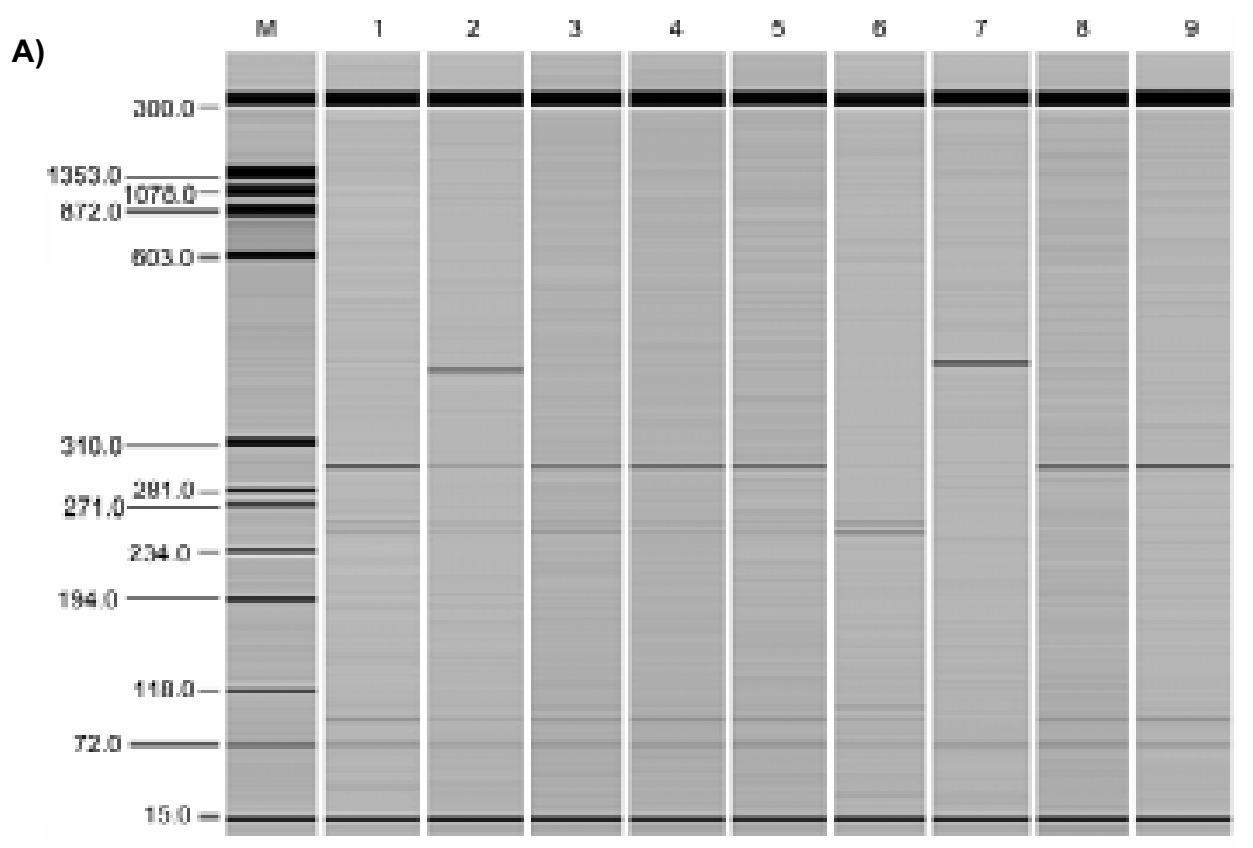

B)

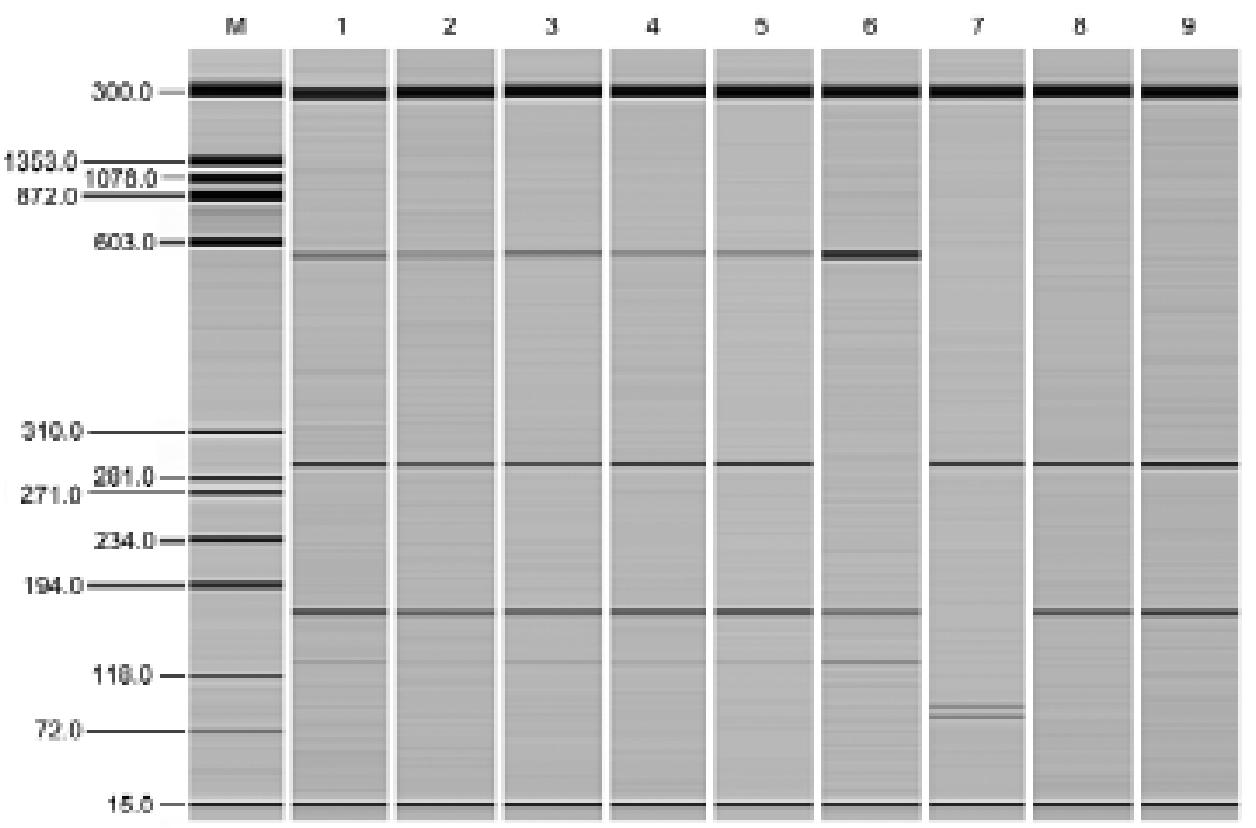

Fig. 1. Electrophoretic gel image of PCR products digested with Hae III (A) and MspI (B). M-QX DNA Size Marker FX 17.

Lines 1-5 strains with genotype C (IHEM19482, 19076, 19265, 19491, 19608), line 6 genotype B (IHEM 19651), line 7 genotype A subtype d (1228, laboratory collection), lines 8 and 9 genotype A subtype a (IHEM 19069, 19144). Performed with the help of Biocalculator software (Qiagen).

for $1 \mathrm{~min}$, and $72^{\circ} \mathrm{C}$ for $4 \mathrm{~min}$, with a final extension at $72^{\circ} \mathrm{C}$ for $4 \mathrm{~min}$ following the last cycle. The PCR products were digested, separately overnight with the enzymes Hae III (BsuRI) and MspI (HpaII, Fermentas), in accordance with the protocol of Karahan and Akar (2005). The undigested and digested PCR products were analyzed by electrophoresis in $4 \%$ agarose gel and visualized in UV after staining with EtBr. The selected DNA samples were additionally analyzed by capillary electrophoresis in the QIAxcel system
(Qiagen) using a QIAxcel DNA High Resolution Kit, the QX Alignment Marker 15-bp/3-kb, and the QX DNA Size Marker FX 174. The tests were performed with the OM500 method and the results were analyzed by Biocalculator software and presented as both simulated bands on gel images and peaks in electrophoregrams.

PCR with the primer pair CA-INT-L/CA-INT-R and DNA of genotype A resulted in a single PCR product of $\sim 460$ bp (McCullough et al., 1999b), 


\begin{tabular}{|c|c|c|c|c|}
\hline $\begin{array}{l}\text { Hae III } \\
\perp\end{array}$ & $\begin{array}{l}\text { hae } 11 \\
\mid \text { Msp I }\end{array}$ & $\begin{array}{c}\text { Hae III Hae } / \prime \prime \\
\text { Hae } \\
\end{array}$ & $\begin{array}{l}\text { Hae III } \\
\perp\end{array}$ & $\perp^{M s p ~ I}$ \\
\hline & 12 & 400 & 1600 & 1800 \\
\hline
\end{tabular}

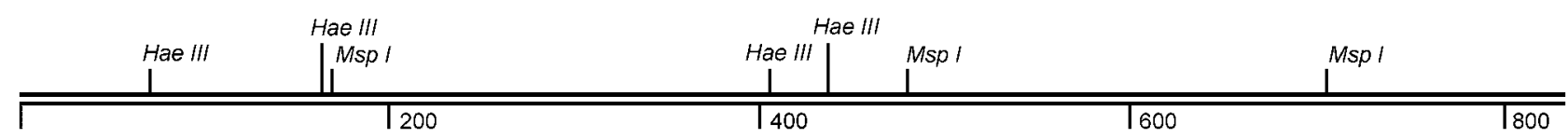

Fig. 2. A diagram presenting the restriction sites of a fragment of sequence DQ465844 (mether line) and the hypothetical sequence in which the intron of DQ465844 was replaced by the X74272 sequence (performed with the help of SeqBilder, Lasergene software).

which can be slightly different in particular subtypes (Karahan and Akar, 2005). The strains of genotype A tested in this study give PCR products of typical size, except for one isolate (no 1228, laboratory collection), which gave a larger product ( $\sim 70 \mathrm{bp}$ ). Most isolates $(61 / 62,98 \%)$ of genotype A showed the same RFLP pattern, namely three fragments of 294, 92, and $72 \mathrm{bp}$, after digestion with Hae III and two fragments of 289 and $171 \mathrm{bp}$ after digestion with MspI (Figs. $1 \mathrm{~A}$ and B). This result corresponds well with "subtype a" described by Karahan and Akar (2005), which was characterized by three bands of 296, 93, and 71 bp after digestion with Hae III. Isolate no 1228 showed a different restriction pattern, consisting of two bands of $\sim 400$ and $72 \mathrm{bp}$ with enzyme Hae III and three bands $(290,93$, and $84 \mathrm{bp})$ with MspI, which is in accordance with the subtype classified as "subtype d" by Karahan and Akar.

In their study, Karahan and Akar tested 144 genotype A isolates obtained from different clinical samples from three Turkish hospitals and $52 \%$ of them were identified as "subtype a", whereas the other strains were distributed among seven different subtypes. In a recently published paper, Gurbuz and Kaleli (2010) found $84(84.8 \%)$ "subtype a" samples among 99 genotype A isolates. The genotype A isolates tested in this study were highly homogenous $(98 \%$ with "subtype a"). Analysis of the results obtained by us and by other authors, indicates that the level of diversity of LSU rDNA varies in particular C. albicans populations and may be geographically related. It is interesting that "subtype d" identified in this study, as well as 9 isolates with "subtype d" described by Karahan and Akar (2005), were obtained from blood, which may suggest a high invasiveness of "subtype d". A future study performed with a higher number of invasive and non-invasive isolates can be helpful in verifying such an hypothesis.

In this study we also performed the RFLP analysis of the PCR products obtained for genotypes B and C. The public PubMed database (http://www.ncbi.nlm. nih.gov/sites/entrez?db=pubmed) contains only two sequences (accession nos. DQ465844 and DQ465845) of LSU of C. albicans genotype B which include the DNA fragment flanked by the CA-INT-L and
CA-INT-R primers. The restriction analysis of these sequences performed with the use of SeqBuilder Lasergene software indicated the same pattern with MspI (four bands of 310, 225, 169, and $131 \mathrm{bp}$ ) and two different patterns with Hae III $(398,242,93,71$, $31 \mathrm{bp}$ for DQ465844, and 248, 242, 182, 93, and $71 \mathrm{bp}$ for DQ465845). This finding suggests sequence diversity in the analyzed LSU fragment of genotype B and the usefulness of the Hae III enzyme in its testing. The results obtained experimentally differed from those predicted on the basis of an analysis of the reference sequences. In our study, all the investigated strains with genotype B showed the same PCR product size ( $~ 840 \mathrm{bp})$ and the same restriction pattern with enzymes Hae III (six bands of 251, 239, 101, 90, 68, and $35 \mathrm{bp})$ and $M s p I(540,170$, and $130 \mathrm{bp})$. It is worth noting that there was some discrepancy between the results obtained by capillary electrophoresis (the above pattern) and classical gel electrophoresis, which cannot easily distinguish the bands of $\sim 239,90$, and $35 \mathrm{bp}$ after digestion with Hae III (data not shown). What is interesting is that replacing the intron present in DQ465844 by the sequence of the intron from another strain (accession no X74272.1) resulted in changing the restriction pattern to one more similar to our finding for genotype B, namely seven bands of 247, 242, 103, 93, 72, 32, and $20 \mathrm{bp}$ for Hae III and three bands of 539, 170, and $131 \mathrm{bp}$ for MspI (Fig 1B). An analysis of the DQ465844 and DQ465845 sequences performed after excluding the intron indicated their high similarity (99.8\%) to "subtype a". This suggests that the strains with genotype B tested in this study can also be related to "subtype a" and their intron to the X74272.1 sequence.

Genotype $\mathrm{C}$ generates two amplicons, one of $\sim 460 \mathrm{bp}$ and one of $\sim 840 \mathrm{bp}$, so the PCR-RFLP result could reflect the sequence diversity of the two products. The five strains of genotype $C$ tested in this study displayed the typical sizes of the PCR products and the same restriction pattern with $M s p I$ (four bands of 580, 290, 170, and $130 \mathrm{bp}$ ), whereas with Hae III, four strains showed the same pattern (five bands of 290, 251, 239, 90, and $70 \mathrm{bp}$ ), but the fifth differed from them by the presence of an additional band of $\sim 400$ bp (Fig. 1A). The evolutionary processes which 
resulted in the formation of the heteroallelic genotype $\mathrm{C}$ were discussed by many authors. McCullough et al. (1999) proposed two hypotheses of genotype C development. The first assumes that genotype $\mathrm{C}$ arises after losing the intron by genotype $\mathrm{B}$ and the second is that genotype $\mathrm{C}$ is formed due to the acquisition of the intron by genotype $\mathrm{A}$, most probably as a result of sexual recombination, which has not yet been detected in C. albicans. Recently, Miletti-Gonzalez and Leibowitz (2008) studied the genetic arrangement of the CaLSU intron in the rDNA of an isolate with genotype $\mathrm{C}$ and showed that intron-possessing rDNA and intron-less rDNA copies are arrayed in tandem and adjacent to each other, forming rDNA clusters present in two R chromosomes. The authors observed high variability in the number of rDNA complex copies among clones of genotype C. The simple RFLP experiment performed in this study showed that the diversity of genotype $\mathrm{C}$ described above can be wider due to variability in the rDNA sequence.

In summary, the presented results indicate a high homogeneity of the analyzed fragment of LSU rDNA in the clinical isolates of $C$. albicans, in contrast to published data obtained with a Turkish collection of yeasts. The restriction analysis of the amplicons obtained after $\mathrm{A}, \mathrm{B}$, and $\mathrm{C}$ genotyping is a simple and reproducible method enabling broader strain characteristics and can be useful in epidemiological and evolutionary studies.

\section{Acknowledgments}

This study was supported by the Polish Ministry of Science and Higher Education ( $\mathrm{Nr} 402055$ 31/1808)

\section{Literature}

Girish Kumar C.P., A. M. Hanafy, M. Katsu, Y. Mikami and T. Menon. 2006. Molecular analysis and susceptibility profiling of Candida albicans isolates from immunocompromised patients in South India. Mycopathologia 161: 153-159.

Gurbuz M. and I. Kaleli. 2010. Molecular analysis of Candida albicans isolates from clinical specimens. Mycopathologia 169: 261-267.

Jayaguru P. and M. Raghunathan. 2007. Group I intron renders differential susceptibility of Candida albicans to bleomycin. Mol. Biol. Rep. 34: 11-17.
Karahan Z.C. and N. Akar. 2005. Subtypes of genotype A Candida albicans isolates determined by restriction endonuclease and sequence analyses. Microbiol. Res. 160: 361-366.

Karahan Z.C., H. Guriz, H. Agirbasli, N. Balaban, J.S. Gocmen, D. Aysev and N. Akar. 2004. Genotype distribution of Candida albicans isolates by $25 \mathrm{~S}$ intron analysis with regard to invasiveness. Mycoses. 47: 465-469.

McCullough M.J., K.V. Clemons and D.A. Stevens. 1999a. Molecular epidemiology of the global and temporal diversity of Candida albicans. Clin. Infect. Dis. 29: 1220-1225.

McCullough M.J., K.V. Clemons and D.A. Stevens. 1999b. Molecular and phenotypic characterisation of genotypic Candida albicans subgroups and comparison with Candida dubliniensis and Candida stellatoidea. J. Clin. Microbiol. 37: 417-421.

Mercure S., S. Montplaisir and G. Lemay. 1993. Correlation between the presence of a self-splicing intron in the 25S rDNA of C. albicans and strains susceptibility to 5-fluorocytosine. Nucleic. Acids. Res. 21: 6020-6027.

Miletti-González K.E. and M.J. Leibowitz. 2008. Molecular characterization of two types of rDNA units in a single strain of Candida albicans. J. Eukaryot. Microbiol. 55: 522-529.

Millar B.C., J. Xu, R. McMullan, M.J. Walker, S. Hedderwick and J.E. Moore. 2005. Frequency and distribution of group I intron genotypes of Candida albicans colonizing critically ill patients. Br. J. Biomed. Sci. 62: 24-27.

Millar B.C., J.E. Moore, J. Xu, M.J. Walker, S. Hedderwick and R. McMullan. 2002. Genotypic subgrouping of clinical isolates of Candida albicans and Candida dubliniensis by $25 \mathrm{~S}$ intron analysis. Lett. Appl. Microbiol. 35: 102-106.

Nawrot U, J. Skala, A. Noczynska, N. Potocka, K. Koczocik and E. Baran. 2004. Distribution of Ca.LSU intron and acid protease production by Candida albicans strains isolated from gastrointestinal tract of diabetes children. Pol. J. Microbiol. 53: 189-191. Nawrot U., J. Skała, K. Włodarczyk, P-A Fonteyne, N. Nolard, J. Nowicka. 2008. Proteolytic activity of clinical Candida albicans isolates in relation to genotype and strain source. Pol. J. Microbiol. 57: 27-33.

O'Donnel K., E. Cigelnik, N.S. Weber and J.M. Trappe. 1997. Phylogenetic relationships among ascomycetous truffles and the true and false morels inferred from $18 \mathrm{~S}$ and $28 \mathrm{~S}$ ribosomal DNA sequence analysis. Mycologia 89: 48-65.

Qi Q.G., T. Hu and X.D. Zhou. 2005. Frequency, species and molecular characterization of oral Candida in hosts of different age in China. J. Oral. Pathol. Med. 34: 352-356.

Tamura M., K. Watanabe, Y. Mikami, K. Yazawa and K. Nishimura. 2001. Molecular characterization of new clinical isolates of $C$. albicans and C. dubliniensis in Japan: analysis reveals a new genotype of $C$. albicans with group I intron. J. Clin. Microbiol. 39: 4309-4315.

Zhang Y., Z. Li, D.S. Pilch and M.J. Leibowitz. 2002. Pentamidine inhibits catalytic activity of group I intron Ca.LSU by altering RNA folding. Nucleic. Acids. Res. 30: 2961-2971. 